\title{
EL ENSAYO ARS MEDICA DE JOHN LOCKE, Y LA INFLUENCIA DE SUS IDEAS MÉDICAS SOBRE LA FILOSOFÍA EMPIRISTA
}

\author{
Miguel Ángel Sánchez González \\ Unidad de Historia de la Medicina. Facultad de Medicina. Universidad Complutense de Madrid. \\ migsan@ucm.es
}

Recibido: 12 diciembre 2012; Aceptado: 2 junio 2013.

Cómo citar este artículo/Citation: Sánchez González, Miguel Ángel (2014), “El ensayo Ars Medica de John Locke, y la influencia de sus ideas médicas sobre la filosofía empirista", Asclepio 66 (1): p39. doi: http://dx.doi.org/10.3989/asclepio.2014.13

RESUMEN: Se presenta, analiza y traduce por primera vez al castellano, el ensayo Ars Medica escrito por John Locke en 1669. Este ensayo muestra los problemas médicos que dieron origen al conjunto de la filosofía de Locke; y ofrece claves esenciales para comprender los motivos, las metas y los rasgos de la teoría del conocimiento empirista. Ideas originariamente médicas que tuvieron una repercusión directa en la obra posterior de Locke fueron: 1) Confianza en la perfectibilidad de la ciencia y elucidación del papel que la reflexión filosófica podía desempeñar en ello. 2) Rechazo del conocimiento deducido de principios, hipótesis o máximas. 3) El método "histórico simple» para edificar el conocimiento; que intentaba registrar los hechos descriptivamente, renunciando al estudio de las causas últimas. 4) Una clasificación tripartita de las ciencias que instaura la semiótica, ciencia médica por antonomasia, y la define como un mero conocimiento de los signos de las cosas, que no pretende discernir las esencias ni las sustancias. Locke, en su epistemología, generaliza para todo el conocimiento científico la teoría médica de los signos de las enfermedades. Convierte todo conocimiento posible se en mero saber descriptivo de signos. $Y$ atribuye al conocimiento un carácter probabilístico y pragmático.

PALABRAS CLAVE: Empirismo; Medicina de Sydenham; Semiótica; Epistemología; Certeza y Probabilidad de la Ciencia.

\section{THE JOHN LOCKE'S ARS MEDICA ESSAY, AND THE INFLUENCE OF HIS MEDICAL IDEAS ON THE EMPIRICAL PHILOSOPHY}

ABSTRACT: John Locke's essay Ars medica of 1669, is presented, analyzed and translated into Spanish. This essay shows the medical problems that prompted Locke's philosophy and provides essential clues to understanding the motives, aims and characteristics of the empiricist's theory of knowledge. Ideas originated in medicine that profoundly influenced subsequent Locke's works were: 1) Trust in the perfectibility of science and elucidation of the role that philosophical thinking could play. 2) Rejection of knowledge deduced from principles, hypotheses or maxims. 3) Reliance on a «plain historical method» for the production of knowledge, which tried to register facts descriptively, while abandoning the study of ultimate causes. 4) Use of a three-part classification of the sciences that enthrones semiotics, medical science par excellence, and defines it as a mere knowledge of the signs of things, that is not intended to discern either essences or substances. Locke, in his epistemology, applies to all scientific knowledge the medical theory of the signs of illnesses. He envisions all possible science as a mere descriptive knowledge of signs; and thinks that knowledge is probabilistic, analogous and pragmatic.

KEY WORDS: Empiricism; Sydenham's medicine; Semiotics; Epistemology; Certainty and Probability of Science.

Copyright: () 2014 CSIC. Este es un artículo de acceso abierto distribuido bajo los términos de la licencia Creative Commons Attribution-Non Commercial (by-nc) Spain 3.0. 
El pensamiento filosófico suele construirse generalizando las reflexiones que suscita el estudio de algún campo de la realidad. En su formulación final, las ideas filosóficas intentan tener validez universal. Es decir: pretenden ser también verdaderas en campos de la realidad distintos al que suscitó la reflexión inicial. Según esto, para entender en profundidad a un filósofo, debemos preguntarnos cuál fue su campo concreto de reflexión inicial. Y muchas discrepancias filosóficas podrían explicarse teniendo en cuenta las diferencias entre los campos que motivaron las afirmaciones en conflicto.

Así, en la Edad Moderna, las discrepancias entre las escuelas filosóficas racionalistas y empiristas se deben en gran medida a que sus autores tomaron distintos campos y disciplinas científicas como base para sus reflexiones filosóficas. Los filósofos racionalistas tomaron como material paradigmático de estudio a las ciencias clásicas físico-matemáticas. Mientras que los filósofos empiristas tuvieron in mente las ciencias llamadas «baconianas»: química, magnetismo, calor y electricidad. Era esperable, por tanto, que sus generalizaciones epistemológicas fueran distintas. (Sánchez González, 1986, pp. 159-161).

John Locke (1632-1704) también tuvo un campo de reflexión original, sin el cual su filosofía empirista no puede ser bien entendida. Y ese campo de reflexión fue la medicina de su época. Hasta hace unas décadas no se había tenido suficientemente en cuenta la profesión médica de John Locke, por considerarla inoperante o marginal. Pero hoy sabemos que Locke, durante toda su vida, fue médico por vocación, por dedicación y por conocimientos. Desde el primer año de su llegada como estudiante a la Universidad de Oxford en 1652 comenzó a rellenar libros de notas médicas con toda clase de información disponible a través de sus lecturas, contactos personales y observaciones propias. Inicialmente se graduó como Masters of Arts, en 1658. En 1660 fue nombrado Lecturer de griego, y en 1663 Censor de Filosofía moral. Pero desde el año de su graduación en 1658 había continuado realizando estudios informales de medicina en Oxford como Senior Student. Hasta el punto de que en 1666, transcurridos los siete años preceptivos, se consideró capacitado para optar simultáneamente a los títulos de Bachelor y Doctor of Medicine. Sin embargo, aunque su propuesta fue aceptada por el Chancelor, Locke desistió de presentar su solicitud en ese momento, temiendo el rechazo de algunos profesores. En 1670 realizó un segundo intento de acumular ambos títulos médicos, pero nuevamente se encontró con la oposición de algunas autoridades. Hasta que por fin, en 1675 , en su tercera tentativa de titulación académica consiguió su graduación como Bachelor of Medicine por la Universidad de Oxford. Por entonces tuvo que contentarse con un puesto de medical studentship. En
1678 estuvo a punto de conseguir la cátedra de medicina del Gresham College, pero finalmente, le dieron el puesto a otro. Y John Locke nunca llegaría a conseguir el grado de Doctor of Medicine.

Lo cierto es que John Locke siempre se consideró a sí mismo como médico. Desde 1666 ganó su sustento como médico y secretario personal de Lord Ashley, un conocido aristócrata, líder del partido Whig, que más tarde llegaría a ser el primer Earl of Shaftesbury. Pero el aspecto médico más importante de la vida de Locke fue su relación personal con el Dr. Thomas Sydenham, con quien trabajó conjuntamente desde 1667 hasta 1675. Posteriormente, nunca perdió su interés por la medicina, y nunca dejó de actuar ocasionalmente como consultor médico. Pero su trabajo al servicio de Lord Ashley le obligó a diversificar sus esfuerzos y dedicarse a los más variados temas. De modo que, al final de su vida, lamentó no haber podido dedicarse más completa y exclusivamente a la medicina. Así lo confiesa en una carta dirigida a un amigo:

si yo hubiera empleado esos años en los que viví con él [Lord Ashley, recientemente fallecido] en la práctica pública de la medicina creo que puedo decir sin alardear que me hubiera podido procurar a mí mismo otra forma de establecimiento que la que tengo ahora; [...] Yo, en los últimos e infelices tiempos me dediqué al estudio de la medicina. Pero, iDios mío!, un accidente de mi vida (el ir a parar entre la familia de un gran hombre) y una falla en mi salud (una indisposición consuntiva que me impidió un asentamiento sosegado para cuidar de la salud de otros y me dio bastante trabajo en cuidar de mí mismo), han confundido (yo no sé por qué brujería) el sosiego que yo siempre busqué ${ }^{1}$.

Teniendo en cuenta todos estos antecedentes, la epistemología de John Locke puede ser vista como una generalización de perspectivas y problemas que inicialmente fueron médicos. Y sus propuestas sobre el método científico fueron sobre todo un intento de justificar y promover el método que el doctor Thomas Sydenham estaba aplicando a la medicina. Ese método, originalmente médico, fue luego generalizado y propuesto por el filósofo como un instrumento para la elaboración de todas las ciencias naturales (Romanell, 1984, y Sánchez González, 1987).

Recordemos la declaración del propio Locke sobre los problemas que dieron origen a su Ensayo sobre el entendimiento humano: "Estando reunidos cinco o seis amigos en mi despacho, y conversando sobre un tema muy alejado de éste, pronto nos vimos en un punto muerto [...] llegué a pensar que habíamos tomado un camino equivocado; y que antes que nos dedicáramos a las indagaciones de esa naturaleza, era necesario examinar nuestras propias capacidades, y ver cuáles eran los objetos para cuyo trato era o no adecuado nuestro entendimiento [...]. Algunos 
pensamientos apresurados y mal digeridos sobre un tema que nunca había yo considerado antes, y que redacté para nuestra siguiente reunión, dieron la primera entrada a este tratado ${ }^{2}$.

Locke omitió mencionar cuál fue el tema concreto que motivó aquella discusión inicial. No obstante, la crítica filosófica ha creído tradicionalmente que el Ensayo fue motivado por problemas de índole teológico y moral. Pero, en la segunda mitad del siglo XX, Patrick Romanell ofreció argumentos convincentes para pensar que el problema inicial que motivó el Ensayo fue de carácter médico (Romanell, 1958). Y también, en la copiosa correspondencia que mantuvo John Locke pueden encontrarse indicios de que él mismo no consideraba su obra como una respuesta a interrogantes de orden ético-religioso. Baste citar la siguiente carta, escrita por Locke en 1697: «Hace siete años que se ha publicado este libro [el Ensayo sobre el entendimiento humano]. Después de un silencio de cinco o seis años se comienza a descubrir allí no sé yo qué defectos de los que no se habían apercibido antes; y, lo que es más singular, se pretende encontrar materia para controversias de Religión en esta obra donde yo no he tenido la intención de tratar más que cuestiones de pura especulación filosófica» ${ }^{3}$.

Así pues, no parece adecuado imaginar que el famoso Ensayo tuvo su origen en una preocupación teológica. $\mathrm{Y}$ el silencio de Locke sobre el tema de su reunión inicial debe interpretarse como una prueba más de su afán de generalizar al conjunto de la ciencia unas premisas que fueron primordialmente médicas.

Algunos aspectos de la filosofía de John Locke en los que puede detectarse la influencia de la medicina de su época fueron los siguientes (Sánchez González, 1990):

Su descripción y recomendación entusiasta del llamado «método histórico simple» para la construcción de la ciencia. Un método que había sido usado por Sydenham para confeccionar sus historias clínicas.

Su énfasis en la observación y la experiencia sensorial para sustituir la búsqueda de las esencias de las cosas, tal y como recomendaba la medicina sydenhamiana.

Su rechazo de las hipótesis y de los principios.

Su negativa a investigar las causas últimas y los mecanismos íntimos de los acontecimientos.

Su ideal de evidencia sensorial irrefutable.

Su escepticismo sobre las posibilidades de certeza de la ciencia.

Por todo lo cual, la lectura de los ensayos médicos de John Locke puede resultar iluminadora; precisamente porque en ellos encontramos muchas claves interpretativas de su pensamiento filosófico.

\section{EL ENSAYO ARS MEDICA, ESCRITO EN 1669}

En 1669 escribió Ars Medica, un ensayo en el que analizaba los fundamentos de la medicina y el método para perfeccionarla. Nunca llegó a publicarse en vida del autor, y se ha conservado entre los manuscritos de John Locke en el Public Record Office de Londres ${ }^{4}$.

El período de 1666 a 1670 fue para Locke su etapa de mayor dedicación a la medicina práctica, en contacto con el Dr. Sydenham. Y, como ya hizo constar su biógrafo Fox Bourne, Locke: «Estaba ahora aplicando a las cuestiones médicas más urgentes el mismo criterio de verdad y utilidad que luego iba a aplicar a las cuestiones metafísicas en su Ensayo sobre el entendimiento humano" (Fox Bourne, 1969, p. 222). Efectivamente, poco tiempo después comenzó a escribir su monumental Ensayo, cuyo manuscrito "A» lleva la fecha de $1671^{5}$.

En la redacción de Ars Medica manifiesta un estilo descuidado, con abundantes correcciones e intercalaciones. Y el escrito queda abruptamente interrumpido en un cierto punto. Tiene pues el carácter de un manuscrito previo para un escrito médico más largo que nunca llegó a escribir.

Kenneth Dewhurst puso en duda la autoría de John Locke, y pensó que este borrador expresa más bien las ideas del Dr. Thomas Sydenham (Dewhurst, 1966, p. 73). Otros autores, por el contrario, han opinado que este ensayo fue algo más que una mera copia o reproducción de las ideas de Sydenham.

Los argumentos que inclinan a atribuir la autoría intelectual al famoso filósofo son los siguientes. En primer lugar, es cierto que Locke copiaba a menudo en sus cuadernos los textos o las ideas de otros autores; pero casi siempre que actuaba así añadía al final del texto copiado el nombre o las iniciales de su verdadero autor, y esto no lo hizo en este ensayo. Por otra parte, no se ha encontrado ninguna otra obra de Sydenham que resulte lo bastante superponible a este fragmento. Además, François Duchesneau (1973, pp. 47-59 y 68-83) ha encontrado diferencias entre la filosofía de la Medicina que se expresa en el fragmento en cuestión y la filosofía médica que se deduce del resto de las obras de Sydenham. Debido a éstas y otras razones, Patrick Romanell (1984, p. 50), incluyó ya el fragmento Arte Médica entre las obras de Locke. Guy Meynell (1994) sugirió que tanto este fragmento como el de Anatomía (Sánchez González, 1988 y 2009), fueron concebidos como borradores para un prefacio a las Observationes medicae que Thomas Sydenham publicaría en 1676. El mismo Meynell (2006) considera incluso el prefacio a dichas Observationes como una obra de colaboración entre Locke y Sydenham, basándose en las similitudes casi literales que pueden reconocerse entre el citado 
prefacio y los fragmentos de Locke. Asimismo Jonathan Walmsley (1998) ofreció argumentos a favor de la autoría de Locke. Y finalmente Anstey y Burrows (2009), aplicando a los textos las últimas técnicas de estilística computacional, han suministrado pruebas, al parecer definitivas, para considerar a Locke como autor del fragmento.

En cualquier caso, la compenetración conceptual entre Locke y Sydenham en materias de medicina fue tanta que podemos considerar este ensayo sobre el Arte Médica como un tratado característico de aquella medicina sydenhamiana que John Locke aprobó y defendió sin reservas.

Locke manifestó repetidamente su entusiasta aprobación del método observacional de Sydenham; como por ejemplo, en esta carta al doctor Thomas Molyneux escrita tras la muerte del maestro de la medicina: «Espero que esta época tenga muchos que sigan su ejemplo, y por la vía de las observaciones prácticas exactas, tal y como él [Sydenham] ha comenzado tan felizmente, incrementen la historia de las enfermedades, y perfeccionen el arte de la Medicina» ${ }^{6}$.

Lo interesante del fragmento Ars Medica es que no podemos dudar de que Locke suscribió plenamente las ideas que expone. Hasta el punto de convertirse en lo sucesivo en su portavoz y defensor sin reservas.

El propósito declarado de este escrito es el de proponer los asuntos médicos a la consideración de los sabios, para incitarlos a la mutua asistencia en el perfeccionamiento de este arte y al establecimiento de una práctica segura en la cura de las enfermedades. Empeños éstos que, como dice John Locke, superan la capacidad de un hombre solo. Nuestro médico filósofo se muestra esperanzado de que la medicina es capaz de grandes adiciones, con tal que se siga un método algo diferente del tradicionalmente usado. Exige este método renunciar a las vanas especulaciones sobre hipótesis y teorías sistemáticas, para concentrarse en la observación y la experiencia propia. Piensa John Locke que la "gran fábrica del mundo» no puede ser comprendida perfectamente en sus causas ocultas y sus mecanismos íntimos. Pero la observación minuciosa y los experimentos prácticos bien diseñados pueden ofrecernos invenciones útiles para las conveniencias de la vida humana.

Todo el ensayo Ars Medica es una exposición y comentario de la medicina sydenhamiana. Y esta medicina, en esquema, puede ser entendida como una nueva y original síntesis entre la antigua medicina hipocrática y la nueva metodología científica de Francis Bacon. Estas dos corrientes coexisten armónicamente en el ensayo, y pueden ser claramente detectadas. Pero, para nuestro objetivo, interesa más señalar las ideas de Ars medica que tuvieron una repercusión di- recta en la obra posterior de John Locke. Algunas de estas ideas son las siguientes:

1) La confianza en la perfectibilidad de la Ciencia y elucidación del papel que la reflexión filosófica podía desempeñar siempre que se limite a procurar nuestro bienestar material

En general, el optimismo científico fue muy propio del ambiente intelectual de principios y mediados del siglo XVII. Esta fue la época de los grandes alegatos épicos en pro del perfeccionamiento de las ciencias, tal y como pueden encontrarse de modo eminente en la obra de Francis Bacon. Los hombres de principios de aquel siglo albergaban la idea de que la revolución científica podía producirse en una sola generación, o quizá dos. Si bien es cierto que a finales de ese mismo siglo se habían dado cuenta de que sólo se había abierto un camino hacia un futuro ilimitado, y que las ciencias estaban aún en sus comienzos. Pero, todavía en 1678, John Locke manifestaba su confianza en las increíbles realizaciones científicas que consideraba inminentes: "Para mí es seguro que nuestros descendientes han de descubrir muchas cosas que a nosotros no sólo nos son desconocidas sino que hasta nos parecen imposibles; incluso no hay que desesperar de este mismo siglo " ${ }^{7}$. También en su Ars Medica expresa rotundamente esta confianza en el futuro de la ciencia, y la ve confirmada precisamente por los recientes avances que ha experimentado la medicina en los últimos tiempos. Esos hombres de mediados del siglo XVII, cuando buscaban ejemplos de adelantos científicos sobre los que poder fundamentar su optimismo, encontraban frecuentemente esos ejemplos en el seno de la Medicina. Recordemos que, así como la Medicina, desde el Renacimiento, había comenzado a renovarse en todos sus frentes, la obra de Newton todavía no había sido dada a la luz, y por su parte la química sólo ofrecía unos escasos resultados prácticos.

En 1669 John Locke dedica su Ars Medica al análisis del método de la medicina y al de las posibles vías para mejorarlo. Y sólo dos años más tarde elaboró su magno Ensayo sobre el Entendimiento con ese mismo objetivo, pero generalizado ya para el conjunto de la ciencia: "es ya bastante ambición emplearse como un trabajador de base para limpiar un poco el terreno, y quitar algunos de los escombros que permanecen en el camino del conocimiento ${ }^{8}$.

De todas formas, el optimismo científico del siglo XVII nunca fue ilimitado. Y su máxima aspiración fue la de rescatar todo el relativamente pequeño resto de conocimiento accesible al hombre en su actual estado de humana limitación. Dentro de una concepción providencialista, ese máximo grado de conocimiento posible era el permitido por Dios. Y estaba constituido casi exclusivamente por los conocimientos que podían ser de utilidad para satisfacer las necesida- 
des humanas más auténticas. Por eso Locke, en su Ensayo llega a decir: "Podemos tener experimentos y observaciones históricas, de las que podemos extraer ventajas de comodidad y salud, e incrementar por ello nuestro arsenal de utilidades para esta vida; pero más allá de esto temo que nuestros talentos no alcanzan, y tampoco son nuestras facultades, según yo imagino, capaces de progresar ${ }^{9}$.

2) Otra idea de John Locke relacionada con la medicina fue su rechazo del conocimiento deducido de principios, hipótesis o máximas.

Esta idea militaba en contra del antiguo ideal científico de la sistematización deductiva. Este ideal antiguo, de inspiración aristotélica había llegado a su culminación en la geometría de Euclides y en la estática de Arquímedes. Y toda la ciencia antigua, incluyendo la medicina galénica, aspiraba a satisfacer ese ideal deductivo. Según él, los científicos debían descubrir los "principios universales del conocimiento", los cuales podrían usarse después como premisas silogísticas desde las que deducir todos los enunciados científicos. Se obtenían así demostraciones y explicaciones que eran "necesariamente ciertas». Pero la reforma metodológica que llevaron a cabo los científicos modernos, encabezados por Francis Bacon, estuvo encaminada principalmente, a reemplazar ese ideal deductivo. Bacon había dicho: «no se puede permitir que el entendimiento salte y vuele de los casos particulares a los axiomas más remotos y generales (como los llamados principios de las artes y de las cosas) y pruebe y confirme los axiomas intermedios mediante la inmóvil verdad de los mismos ${ }^{10}$. Por eso Robert Boyle tituló su obra principal El químico escéptico, habiendo observado que: "gran parte de los erróneos razonamientos y conclusiones provienen de asumir principios falsos o inseguros de los que extraer consecuencias» ${ }^{11}$. Y aún el propio Isaac Newton adoptó como lema: Hypotheses non fingo [no imagino hipótesis]. Sobre estos precedentes se inserta el rechazo de John Locke a los principios generales porque, "en lugar de ser conducidos hacia la verdad a través de los principios, sólo seremos confirmados en la equivocación y el error» ${ }^{12}$.

Ahora bien, en el caso de Locke ese rechazo tenía motivos añadidos que eran específicamente médicos. $Y$ es que entre todas las ciencias, la Medicina era la que más se había resistido siempre a la sistematización deductiva. Ya en el Corpus hipocraticum, escrito dos mil años antes, se había denunciado que: "Los que fabrican estos sistemas y desvían la medicina en la dirección de las hipótesis, lejos del verdadero camino, no sé cómo podrán tratar a los enfermos de acuerdo con sus principios» ${ }^{13}$. Sydenham, por su parte, fue conocido como "el Hipócrates inglés» por la reconocida semejanza entre la manera de proce- der de los dos médicos. Lo cierto es que Sydenham no ahorra elogios a Hipócrates y le muestra como un ejemplo. En este sentido dijo, por ejemplo, que: «el excelente Hipócrates llegó hasta la cima de la Medicina y estableció los sólidos fundamentos para edificar este arte [...] él mostró plenamente los fenómenos de cada enfermedad sin forzar ninguna hipótesis para su servicio ${ }^{14}$. En la misma línea, el ensayo Ars Medica de John Locke expresa un decidido rechazo por a la edificación de la medicina sobre principios generales preestablecidos. Más tarde, en el Ensayo sobre el entendimiento humano, Locke manifestará idéntico rechazo no limitado ya a la medicina sino referido a la totalidad del conocimiento. Podemos, por tanto suponer que el camino intelectual seguido por nuestro autor fue la generalización de sus argumentos desde el caso particular de la medicina hasta la totalidad del conocimiento científico.

3) El método "histórico simple» para la edificación del conocimiento, que renuncia al estudio de las causas últimas.

John Locke dijo haber empleado un «método histórico simple» (plain historical method) en la elaboración de su teoría del conocimiento. En su célebre Ensayo declaró que: "con este método histórico simple puedo dar alguna relación de las vías por las que nuestros entendimientos llegan a alcanzar esas nociones que tenemos de las cosas, y puedo establecer algunas medidas de la certeza de nuestro conocimiento ${ }^{15}$.

Cuando Locke habla de un método "histórico», se está refiriendo a una forma de registro de los hechos que es meramente observacional, descriptiva, intersubjetiva y no interpretativa. Este concepto de "historia» podía incluir el inventario de todos los fenómenos observables en la Naturaleza. Se inspiraba en las Ilamadas "Historias Naturales» que tan intenso cultivo tuvieron en los siglos XVI y XVII. Pero, sobre todo, tomaba ejemplo de las «Historias Clínicas» descriptivas de casos médicos cuya publicación se incrementó durante esa misma época (Laín Entralgo, 1961, pp.82-88).

Francis Bacon había consagrado un capítulo de su Gran Restauración a la «preparación para la Historia Natural», partiendo de la idea de que: «ningún progreso digno del espíritu humano se hubiera podido realizar o podría realizarse en el futuro en el ámbito de la Filosofía y de las ciencias si faltara una Historia Natural y Experimental ${ }^{16}$.

En tiempos de Locke, el método histórico estaba consagrado en todos los campos de la Filosofía Natural. Pero la Medicina y la Química Médica fueron las ciencias naturales mejor conocidas por nuestro autor. Locke, por tanto, aprendió y se ejercitó en el método histórico a través de estas dos ciencias. La medicina, 
contrariamente a como había sido conceptuada en otros tiempos o en otras escuelas, era entendida y practicada por Sydenham y por Locke como una ciencia plenamente histórico-natural. Así se presenta, por ejemplo, el tratado sydenhamiano escrito en 1669 sobre la viruela y manuscrito por el propio Locke: «Esta es la historia natural de la viruela que comprende los fenómenos verdaderos y genuinos que le pertenecen, tal y como son en su propia naturaleza ${ }^{17}$. Para describir este método histórico podemos utilizar las palabras del propio Locke en una de sus cartas al médico Thomas Molyneux: «Observar con meticulosidad la historia de las enfermedades en todos sus cambios y circunstancias, es un trabajo de tiempo, precisión, atención y juicio; y en donde si los hombres se equivocan, por vanidad o negligencia, pueden convencerse de su error por la naturaleza inequívoca y los hechos materiales (matter of fact), los cuales dejan poco espacio para las sutilezas y las disputas verbales que tanto se utilizan en lugar del conocimiento ${ }^{18}$. Comparemos el método así descrito con la siguiente declaración epistolar de John Locke sobre la forma en que él llevó a cabo su examen del entendimiento humano: «Estando resuelto a examinar el entendimiento humano y las formas de nuestro conocimiento, no por la opinión de otros sino por lo que yo pudiera recolectar por mí mismo desde mis propias observaciones, he evitado expresamente la lectura de todos los libros que trataran del tema en alguna forma, para poder así no tener nada que me predispusiera de ninguna manera, y poder dejar a mi pensamiento libre para emprender sólo lo que la materia por sí misma sugiriera a mis meditaciones ${ }^{19}$.

Además el método histórico de Locke es «simple». Como él mismo aclaró en otra de sus cartas, había elaborado: "un tratado escrito en un estilo simple y popular, que no teniendo en él nada del aire de los instruidos y tampoco mucho del lenguaje de las escuelas, era poco adecuado para el uso o el esparcimiento de aquellos que, como profesores o instructores, se aplicaban a sí mismos a los misterios del conocimiento escolástico». ${ }^{20}$ Así pues, cuando John Locke afirma que su método es simple, está haciendo alusión a su carácter antiespeculativo, y por lo tanto práctico. No olvidemos que este filósofo concebía su propio lugar en la ciencia como el de un removedor de la basura especulativa que habían ido acumulando las "Escuelas». "Ya es ambición suficiente el estar empleado como un obrero subalterno en despejar un poco el terreno y apartar algo de la basura que está situada en el camino hacia el conocimiento ${ }^{21}$. En este sentido los objetivos de De arte medica y del Ensayo sobre el entendimiento humano son coincidentes. De manera que, mientras en la primera obra se intenta apartar la "basura» especulativa del terreno de la Medicina, en la segunda se intenta apartar esa "basura» especulativa de todo el conjunto del conocimiento, que estaría afectado, según Locke de una misma enfermedad especulativa. Y no resulta impropio decir que en John Locke detectó en el conocimiento establecido una "enfermedad especulativa» que era preciso curar. Señalemos, a este respecto, que en el contexto de la medicina de su época siempre se utiliza la palabra "método» para hacer alusión a una terapéutica o methodus medendi. Este es, desde luego, el significado que se le atribuye en la obra de Sydenham quien precisamente tituló su primer libro: Methodus curandi Febres.

Sobre la palabra "método», Donald Bates ha distinguido tres usos distintos durante los siglos XVI y XVII: «como forma de enseñanza o persuasión en la Retórica, cultivado por los Humanistas; como forma de conocimiento o descubrimiento en la Filosofía, cultivado por los hombres de «Escuela; y como actuación o práctica en Medicina, cultivado por los médicos» (Bates, 1977, p. 337). El método del que habla Locke no es el retórico. Y él tampoco se consideraba a sí mismo como un hombre de "Escuela», a quienes atacó repetidamente. Su método conservaba algo del carácter terapéutico que tenía para Sydenham y los médicos de su época. Y debemos pensar que Locke, en su epistemología, habría perseguido un equivalente a lo que hizo Sydenham en Medicina: elaborar una historia natural del conocimiento para prescribirle un método de curación. Si esto es así, el título de su célebre Ensayo, parafraseando a Sydenham, podría haber sido: Methodus curandi intellectum. Porque John Locke se vio a sí mismo diagnosticando una enfermedad del conocimiento de los hombres, y pretendió encontrar un método para su curación. Fue así como interpretaron su trabajo epistemológico algunos de sus amigos más íntimos; a saber: como una labor de diagnóstico y terapéutica de las enfermedades del intelecto. En este sentido, su amigo el Dr. Guenelon le escribió: «El señor le Clerc me ha dicho que está usted trabajando en una nueva obra para descubrir las enfermedades del espíritu, esto es una nueva obligación que el público le deberá. iQué beneficio no se producirá, si usted puede curar a los hombres de sus falsas ideas, y ponerles por medio de un método en el camino de la verdad! $»^{22}$

Ya en 1949 Gunnar Aspelin, hablando de la similitud existente entre las ideas metodológicas de Sydenham y las de Locke, señalaba que ambos autores habían empleado un mismo "método histórico». "Sydenham aplica este método histórico al estudio de las enfermedades y los padecimientos [...] Locke a los cambiantes fenómenos de la mente» (Aspelin, 1949, p. 36). Y, algunos años más tarde, Patrick Romanell afirmaba: «El método histórico simple de Locke es el método histórico natural de la medicina de Sydenham generalizado. Esto es, un método que deliberadamente evita todas las explicaciones de naturaleza especu- 
lativa y aparentemente se restringe a sí mismo a la observación, descripción y clasificación de los hechos. Tal y como Sydenham, siguiendo a Hipócrates y Francis Bacon, se vio a sí mismo como un historiador natural de la enfermedad, así Locke a su vez, siguiendo a Sydenham y a su propia experiencia médica, intenta denodadamente en su Ensayo ser un historiador natural del conocimiento» (Romanell, 1958, p. 316).

Como conclusión podemos afirmar que el método histórico simple es la pieza clave de todo el empirismo lockeano. En su Ensayo sobre el entendimiento humano encontramos abundantes manifestaciones de este método. Más aún, todo su Ensayo puede entenderse como un intento de justificar, fundamentar y promover este mismo método como instrumento para la elaboración de todas las ciencias naturales.

4) La clasificación tripartita de las ciencias, que instaura la semiótica, ciencia médica por antonomasia, y la define como un mero conocimiento de los signos de las cosas, que no pretende discernir las esencias ni las sustancias.

En su Ensayo sobre el entendimiento humano, Locke divide la ciencia en tres grandes clases. La primera sería la Física o Filosofía Natural; la segunda es la Práctica o habilidad de aplicar correctamente nuestros actos, y cuya parte más importante sería la ética. Pero la tercera clase es bautizada por Locke con el significativo nombre de Semiótica, y la define de la siguiente forma: "La tercera rama se puede llamar Semeiotikè o doctrina de los signos; y como los más usuales de los cuales son las palabras, también se le llama con bastante propiedad Logikè, Lógica; cuya materia es la consideración de la naturaleza de los signos de los que la mente hace uso para la comprensión de las cosas o para comunicar su conocimiento a otros ${ }^{23}$. Semejante clasificación tripartita de las ciencias disfrutaba de antecedentes clásicos. Los estoicos habían ya dividido el conocimiento en tres partes: Lógica, Física y Ética. Pero Locke introduce, en primer lugar, la novedad de convertir a la ética en parte de una ciencia más amplia llamada Práctica. En lo cual podemos adivinar un intento de conceder estatuto epistemológico a otras actividades prácticas, entre las que no podría dejar de figurar la terapéutica médica. Pero la segunda novedad introducida por John Locke revela más claramente sus orígenes médicos. Consiste en utilizar el término "semiótica» en lugar del tradicional de "Lógica». Esta elección debe hacernos recordar que en la Antigüedad el término semiótica fue usado para designar la parte de la Medicina que se ocupaba de interpretar los signos de las enfermedades, y que abarcaba la diagnosis y la prognosis. Y en la época de Locke el término en cuestión conservaba su significación médica. Como prueba de ello podemos ver que alguno de los libros médicos que poseía el propio John Locke llevaba el título de semiótica cuando hacía referencia a los signos de las enfermedades. Así, por ejemplo, la obra de Th. Fienus titulada Semiotice (1664), que llevaba como subtítulo: "Tratado de los signos médicos» (Harrison and Laslett, p.135) Y la inspiración médica de la semiótica de Locke es importante porque puede explicar el moderno giro epistemológico que tuvo lugar en la forma de elaborar la semántica. Como señala N. Kretzmann: "Las investigaciones semánticas durante la Edad Media y el Renacimiento se habían vinculado íntimamente a la Lógica y a la Gramática. La nueva orientación epistemológica de la semántica, evidente incluso en los libros de Lógica de la Ilustración, quedó establecida explícitamente por primera vez en el Ensayo de Locke» (Kretzmann, 1981, p. 226). John Locke parecía consciente de esta novedad cuando afirmaba que: "si las ideas y las palabras fueran sopesadas distintamente y consideradas debidamente nos proporcionarían una clase de lógica y de crítica distinta de aquélla con la que hasta ahora hemos estado familiarizados ${ }^{24}$. Según la doctrina semiótica de John Locke los instrumentos del conocimiento, que son las ideas y las palabras, no son más que signos directos o indirectos de las cosas. Sin que sea posible ningún conocimiento inmediato de las cosas en sí mismas. De este modo, todo conocimiento posible consiste en un mero conocimiento de signos. Ahora bien, entender así todo el conocimiento humano puede verse como una generalización del caso particular en el que se encuentra el diagnóstico médico. Porque lo único que puede conocer el médico son los signos y síntomas externos indicativos de las distintas enfermedades. Además, para la medicina de Sydenham, las enfermedades son incognoscibles en su esencia. Sydenham decía: "La esencia de esta enfermedad no pretendo definirla con precisión; [...]. La Naturaleza lleva a cabo la generación de todas las cosas; y aun cuando saca muchas cosas desde el abismo de la causa hasta la clara luz del efecto, deja veladas en la más profunda oscuridad sus esencias, sus diferencias constitutivas y sus naturalezas» ${ }^{25}$. Locke, al declarar que todas las esencias son incognoscibles, no hace sino generalizar para todo conocimiento posible el postulado médico de Sydenham sobre la imposibilidad de conocer la esencia de las especies morbosas: "Las esencias reales no las conocemos. Nuestras facultades no nos llevan más allá, en el conocimiento y distinción de las sustancias, que a una colección de aquellas ideas sensibles que observamos en ellas ${ }^{26}$. Es decir, que todos los objetos del Universo, al igual que ocurría con la especies morbosas de Sydenham, sólo pueden conocerse por sus respectivos signos sensibles. No obstante, sigue siendo posible elaborar una ciencia de los signos, esto es, una semiótica. Aunque ese conocimiento, al no estar fundado sobre la verdadera naturaleza de las cosas, sólo podrá llegar a tener un carácter probabilístico. Y curiosamente, Locke explica el carácter 
probabilístico del conocimiento con argumentos que podría haber hecho suyos cualquier médico de su época: «en asuntos de probabilidad [...]. ¿Quién hay que haya tenido el ocio, paciencia y medios para reunir todas las pruebas y así concluir con seguridad que posee una visión clara y completa, y que no hay nada más que se podría agregar para mejorar su información? Y sin embargo estamos obligados a determinarnos en un sentido o en otro ${ }^{27}$. Oigamos a Sydenham expresar unas reflexiones semejantes: «Estoy lejos de atribuirme el mérito de haber agotado mi tema con las presentes observaciones. Es altamente probable que yo me haya equivocado incluso en la enumeración completa de las epidemias» ${ }^{28}$. Así pues, el carácter probabilístico acompaña siempre al juicio médico. No ocurre igual con el conocimiento del matemático o el del físico. Por eso, cuando Locke implantó una concepción probabilista del conocimiento, tuvo que sentirse inspirado fundamentalmente por el modelo de las ciencias médicas.

Además, la semiótica lockeana hace un hincapié desconocido hasta entonces en la investigación de la naturaleza del error y de las causas de nuestra ignorancia. Porque como él dijo: «Si miramos un poco el lado oscuro y echamos una mirada a nuestra ignorancia: la cual, siendo infinitamente más amplia que nuestro conocimiento, puede servirnos mucho para acallar las disputas y mejorar el conocimiento útil» ${ }^{29}$. La lógica clásica anterior se había presentado más bien como un sistema garantizador de la verdad de la verdad de las proposiciones. Pero Locke no indagará prioritariamente en los criterios indicativos del conocimiento verdadero sino en los síntomas reveladores de nuestra ignorancia. De esta forma, pretendiendo descubrir las manifestaciones de nuestra ignorancia, Locke está actuando en analogía con el médico cuya labor es la de revelar los síntomas de las enfermedades. Y al actuar así, también puede decirse que está concibiendo el error intelectual como el contrapunto psíquico de la enfermedad física.

También debió tener inspiración médica la importancia que Locke concedió al método de la analogía para la edificación del conocimiento, especialmente en el caso de causas y mecanismos ni directamente accesibles a los sentidos. "La analogía en estos asuntos es la única ayuda que tenemos, y solamente en ella fundamos todos los cimientos de la probabilidad [...], y un cauteloso razonamiento a partir de la analogía nos conduce a menudo al descubrimiento de verdades y producciones útiles que de otro modo permanecerían ocultas $»^{30}$. Tenemos en esto un nuevo argumento para defender el trasfondo médico de la semiótica de Locke. Puesto que la analogía es un antiguo método de edificación del conocimiento médico. Tal y como L. Edelstein señaló: «Las investigaciones de los médicos presocráticos sobre los órga- nos internos del cuerpo humano se basaban en analogías. También los médicos hipocráticos explicaron lo desconocido por comparación con lo conocido» (Edelstein, 1967, p. 292).

Podemos llegar a la conclusión de que la transformación de la lógica en una semiótica de los signos del conocimiento es una trasposición a la epistemología de la teoría médica de los signos de enfermedades. De esta forma el Ensayo sobre el entendimiento humano, ateniéndonos a la clasificación del propio Locke, debe ser considerado como un tratado de semiótica. Y podemos afirmar que John Locke concibió su tarea como lógico en analogía con la del médico diagnosticador y curador de enfermedades.

\section{ARS MEDICA $1669^{31}$}

La prolongación de la vida, libre de enfermedad y dolor, tanto como sea capaz la constitución de nuestra frágil contextura, es una preocupación tan importante para el género humano que apenas se puede encontrar un empeño más grande que la profesión de curar enfermedades; ni hay otro arte que merezca tanto todo el cuidado, la industria y la observación de sus profesores para mejorarlo y llevarlo a la perfección, la cual yo no dudo que es posible en muchas partes y hasta un alto grado. Aquél que se lance a hacer esto merecerá sin duda el agradecimiento del género humano por tener una intención tan buena como es la de someter a certeza esas reglas y métodos de cuya práctica depende el bienestar y la recuperación de los enfermos; pero cualquiera que piense abarcarlo en soledad se encontrará a sí mismo empeñado en un negocio demasiado amplio para la comprensión de cualquier hombre y demasiado grande para su propio esfuerzo solitario. Mi intención es, por tanto, proponer unas pocas cosas a la consideración de los hombres instruidos de esta facultad tan útil, y promover su apoyo mutuo para perfeccionar el arte y asentar una práctica habitual segura en la curación de las enfermedades; para que así, reduciendo cada día el amplio catálogo de las enfermedades todavía incurables y los frecuentes acontecimientos tristes en el resto de ellas (y siendo anulados por el creciente éxito cotidiano de los médicos tanto la falta de confianza que algunos hombres sensatos sobre la base de consideraciones serias parecen tener hacia el arte en sí mismo, como el descrédito que otros procuran atraer artificiosamente sobre la práctica de la medicina), los practicantes de la medicina ingeniosos e instruidos puedan atender su vocación con más confianza y satisfacción, cuando ya no puedan ser amonestados por esos confesables opprobia medicorum que cederán todos los días ante la eficacia de sus medicinas o de sus métodos bien ordenados. Si esto se acometiera alguna vez, quizá este propósito no se encontraría tan imposible como se imagina a primera vista; y el gran mejora- 
miento que algunas partes de la medicina han recibido en estos pocos años me da confianza para creer que aún es capaz de grandes adiciones, y ello de una manera algo diferente a la que hasta ahora parece haber sido seguida generalmente por la mayoría de aquellos que han sido tan benévolos como para propagar el conocimiento de la medicina y dejar las reglas de la práctica a la posteridad, tal y como aparecerá a cualquiera que examine cuidadosamente sus escritos, por los cuales aún debe estar muy agradecida la posteridad; y no se les puede censurar porque ellos hicieron algo que es muy agradable al entendimiento humano el cual, no contentándose con observar la operación de la naturaleza y el acontecer de las cosas, es muy inquisitivo de su causa, y muy impaciente e inquieto hasta que, en aquellas cosas en las que está interesado, forja para sí mismo alguna hipótesis y establece un fundamento desde donde establecer todos sus razonamientos. Por tanto, si los hombres instruidos de las edades previas emplearon una gran parte de su tiempo y sus pensamientos en buscar las causas ocultas de los trastornos, fueron curiosos en imaginar la actividad secreta de la naturaleza y los variados instrumentos imperceptibles con los cuales ella trabaja, y poniendo juntas todas estas fantasías crearon para sí mismos sistemas e hipótesis, no hay que sorprenderse ni censurarles puesto que se acomodaron a las costumbres de sus tiempos y países, y siguieron de esa forma sus inclinaciones más naturales; ahora bien, desear tener alguna base sobre la que apoyar sus pensamientos y algún fundamento para guiarse en la práctica de su arte, mantenerse ocupado y sutil en disputar sobre los principios aceptados, no fue más que estar empeñado en el camino de la fama, la reputación y la ilustración valorados en esa época; y el que su práctica no se haya extendido más allá de lo que permitían los principios sagrados en los que creían no debe sorprendernos más que el hecho de que no hayamos encontrado ninguna edificación justa y duradera legada por nuestros antecesores sobre fundamentos estrechos y poco sólidos. Aquí no se pensará de mí que estoy censurando a los autores instruidos de tiempos pretéritos, o que repudio las ventajas que ellos han dejado a la posteridad. A ellos debemos un gran número de observaciones excelentes y varios discursos ingeniosos, y no hay ninguna regla de práctica fundada sobre observaciones no sesgadas que yo no acoja y sostenga con veneración y agradecimiento: pero aun así yo pienso que puedo afirmar confiadamente que esas hipótesis que hilvanaron los largos y elaborados discursos de los antiguos, y que no se sometieron a indagaciones para extenderlas más allá del cometido de explicar los fenómenos de las enfermedades según las doctrinas y las reglas de la práctica acomodadas a los principios recibidos, no han hecho finalmente más que limitar y estrechar los pensamientos de los hombres, entretener sus entendimien- tos con finas pero inútiles especulaciones, y alejar sus investigaciones del verdadero y ventajoso conocimiento de las cosas. Las nociones que han sido suscitadas en las cabezas de los hombres por principios especulativos remotos, aunque sean verídicas, son como la curiosa imaginería que los hombres ven a veces en las nubes y que se complacen en llamar "los cielos», las cuales, aunque son fantásticas en su mayor parte y en el mejor caso no son más que la contextura accidental de una niebla, realmente perturban la vista y acortan la perspectiva. Y aunque estas apariciones pintadas son suscitadas por el sol y parecen la progenie genuina de la gran fuente de luz, no son realmente nada más que oscuridad y una nube; y cualquiera que viaje con sus ojos fijos en ellas está con una probabilidad de diez contra uno fuera de su camino. Aquél que en Medicina establezca máximas fundamentales y desde allí, extrayendo consecuencias y suscitando disputas, la reduzca a la forma regular de una ciencia, ha hecho en realidad algo que amplía el arte de charlar y ha establecido quizá el fundamento para disputas interminables. Pero si espera llevar a los hombres por medio de tal sistema hacia el conocimiento de las enfermedades de los cuerpos de los hombres, la constitución, naturaleza, signos, cambios e historia de las enfermedades con la forma segura y directa de curarlas, emprende una senda parecida a la del que camina arriba y abajo por un bosque demasiado frondoso cubierto de zarzas y espinas, con el designio de adquirir una panorámica y dibujar un mapa del territorio. Estos teoremas especulativos ofrecen a la medicina un provecho tan pequeño como el que producirían dándolos de alimento a los hombres. Y aquél que piense que llegó a ser experto en las enfermedades estudiando la doctrina de los humores, que las nociones de obstrucciones y putrefacciones le ayudan a curar las fiebres, o que por la familiaridad que tiene con el azufre y el mercurio fue conducido hacia este descubrimiento útil, o que con ciertas medicinas y régimen mata con tanta seguridad en el final tardío de algunas fiebres como cura en otras, puede con la misma racionalidad creer que su cocinero debe su habilidad en asar y hervir a su estudio de los elementos y que sus especulaciones sobre el fuego y el agua le han enseñado que los mismos licores burbujeantes que hierven el huevo hasta endurecerlo ponen a la gallina tierna. El comienzo y el mejoramiento de las artes útiles, y las ayudas de la vida humana, han surgido todos de la industria y la observación; el conocimiento verdadero surgió primero en el mundo por la experiencia y las operaciones racionales; y si este método hubiera sido continuado y todos los pensamientos de los hombres hubieran sido empleados en añadir sus propias pruebas a la observación de otros, la medicina sin duda, así como muchos otros artes, habría estado en una condición mucho mejor que en la que está ahora. Pero el hombre orgulloso, no contento con ese cono- 
cimiento del cual era capaz y que era útil para él, necesita penetrar en las causas ocultas de las cosas, instaura principios y establece máximas para sí mismo sobre las operaciones de la naturaleza, y después espera en vano que la Naturaleza, o en verdad Dios mismo, proceda según esas leyes que sus máximas le habían prescrito. Mientras que sus estrechas y débiles facultades no podrían alcanzar más allá de la observación y la memoria de unos pocos efectos producidos por causas visibles y externas, y aun así de una manera completamente fuera del alcance de su aprehensión, no siendo quizá ningún absurdo pensar que esta grande y curiosa fábrica del mundo, la obra del Todopoderoso, no puede ser comprendida perfectamente por ningún entendimiento excepto por aquél que la hizo, aun así el hombre pretendiendo algo propio de una deidad se afanó en hacer que su imaginación suministrara aquello en lo que le falló su observación, y cuando él no pudo descubrir los principios y las causas y los métodos de la obra de la naturaleza, necesitó crear todo ello sacándolo de su propio pensamiento, y fabricó un mundo para sí mismo, estructurado y gobernado por su propia inteligencia (y así el hombre por deseo de conocer más de lo que era capaz perdió una segunda vez el pequeño resto de conocimiento que le fue dejado). Esta vanidad se extiende a muchas de las partes útiles de la filosofía natural, y ello, cuanto más parecía sutil, sublime o instruido en la misma medida se mostraba pernicioso y dañino, impidiendo el crecimiento del conocimiento práctico. Así, estando empeñados el conjunto de hombres más agudos e ingeniosos en especulaciones vacías por costumbre y educación, el mejoramiento de las artes útiles fue dejado a la clase de gente más humilde que tenía recursos más débiles y menos oportunidades de hacerlo, y fueron por eso mismo calificados con el desgraciado nombre de 'mecánicos'. Por consiguiente llegó a ocurrir que el mundo se llenó de libros y disputas, y los libros se multiplicaron sin incrementar el conocimiento: las generaciones sucesivas crecieron más instruidas sin ser más sabias o más felices, y si por casualidad las conveniencias de la vida humana fueron promovidas por alguna invención nueva, los hombres no fueron conducidos a tales descubrimientos felices por la dirección de especulaciones filosóficas, sino por lo que el azar o los experimentos bien diseñados enseñaban a aquellos que empleaban su tiempo y pensamientos en los trabajos de la naturaleza más que en las máximas de las escuelas. De esto son testigos los labradores, curtidores, herreros, panaderos, tintoreros, pintores, etc. Las grandes invenciones de la pólvora y del imán, que han alterado todos los asuntos del género humano, son ejemplos innegables. Así pues aquellos que habían leído y escrito volúmenes enteros sobre la generación y la corrupción no supieron la forma de preservar o propagar las más humildes especies de criaturas, aquél que podía disputar instruida- mente de nutrición, cocción y asimilación, tenía que observar al cocinero y a la buena ama de casa, para lograr una comida sana y sabrosa, y cualquiera que desease tener jardines hermosos y campos fructíferos, tenía más motivo para consultar la experiencia del labrador torpe y del jardinero iletrado que la del filósofo profundo o el agudo discutidor. Que nadie se ofenda si yo catalogo al cocinero y al granjero junto con el académico y el filósofo. Puesto que hablando aquí del conocimiento de los cuerpos naturales, cuya finalidad y beneficio no puede ser otro que las ventajas y conveniencias de la vida humana, todas las especulaciones sobre este tema por más curiosas o refinadas o aparentemente profundas y sólidas, si no enseñan a sus seguidores a hacer algo mejor, o de una forma más corta y más fácil que como podrían hacerlo sin ellas, o si no les conducen al descubrimiento de algunos inventos nuevos y útiles, no merecen el nombre de conocimiento, y no valen el tiempo derrochado en tales filosofías vacías y ociosas. Aquellos que están ocupados en cultivar y adornar aplicadamente tales nociones secas y estériles se emplean vigorosamente para poca cosa, y por la misma razón, ahora que son hombres, podrían haber retenido los muñecos que hicieron cuando eran niños, e intercambiarlos por esas nociones imprácticas y vacías que no son más que las muñecas de las fantasías e imaginaciones de los hombres, que por bien vestidas que estén, después de cuarenta años de jugar con ellas siguen sin ser más que muñecas, desprovistas de fuerza, empleo o actividad. Pero para no extenderme en el amplio campo de la filosofía natural, donde quizá se implantó primero la fundación del daño, me limitaré en el momento presente, de acuerdo con mi propósito, a esa rama de la misma que se ocupa inmediatamente de la salud de los hombres; y sobre la medicina tomaré en consideración:

1) El presente estado de la facultad de Medicina tal y como ahora se encuentra en lo que atañe a las enfermedades y su curación.

2) Los varios grados y pasos por los cuales creció hasta esa altura a la que ha llegado en el presente, los cuales yo supongo que son estos que siguen: 1 . Experiencia. 2. Método fundado sobre la filosofía y la hipótesis. 3. Botánica. 4. Química. 5. Anatomía. En todo lo cual intentaré mostrar cuánto ha contribuido cada uno al avance del arte de la medicina, y dónde se quedaron cortos para perfeccionarlo.

3) Qué se puede hacer más allá de todo lo anterior, para avanzar hacia una cura de las enfermedades más rápida y segura; es decir, por qué medios y método de práctica de la medicina se puede llevar más cerca de a perfección.

1. Las enfermedades tal y como se presentan bajo el régimen de la medicina, y son más o menos controla- 
das por las aplicaciones y los métodos de ese arte tal y como ahora se encuentra, pueden ser convenientemente divididas en 4 clases. 1 . Aquellas que están casi perfectamente bajo el control de la medicina, y en su mayor parte ceden constantemente a la mano de los médicos hábiles guiados por las reglas establecidas de su arte, y donde ellos pueden a primera vista (en la medida en que es adecuado, con sometimiento a la providencia y al gran dispensador de las vidas de los hombres) emprender la cura con seguridad de tener un desenlace feliz. Aunque no hay que esperar que la enfermedad más humilde deba obedecer siempre a la habilidad del médico más capaz, ni tal vanidad sería tolerable en hombres débiles ignorantes que pretendieran ser los dispensadores de la salud y la vida que son los dones libres del Dios todopoderoso, y aunque su mano incontrolablemente quita u otorga donde le place, aun así lo más común es que él lo haga por la intervención de medios secundarios adecuados; y por consiguiente yo no dudo de que un médico puede en algunos casos con poca presunción asegurar a un hombre enfermo su recuperación, tal como una madre emprende la cura del hambre de su hijo, que también es una enfermedad, pero aun así esto no lo hace él por ningún poder o autoridad suya sobre la naturaleza de las cosas, sino por una correcta aplicación de aquellos remedios que fueron ordenados para la producción de tales efectos, las medicinas correctamente ordenadas siendo tan seguras para recuperar

\section{NOTAS}

1 Locke, John (1976), «Carta a Thomas Herbert, earl of Pembroke", 28-XI-1684: The Correspondence of John Locke, ed. por S. de Beer, Oxford, Clarendon Press, vol. 2, p. 663.

2 Locke, John (1963), "Of Human Understanding: the Epistle to the Reader" en: The Works of John Locke, en 10 vols. Londres, 1823, reimpreso en Aalen, Scientia, vol.1, pp. XLVI-XLVII.

3 Locke (1976). "Carta a Philippus Van Limborch" 29-X-1697: Correspondence, vol. 6, 1981, p. 243.

4 Public Record Office of London, PRO 30/24/47 /2, ff. 47-56

5 La primera edición del llamado Draft $A$ del Essay concerning Human Understanding fue publicada por R.I. Aaron y Jocelyn Gibb en An early draft of Locke's Essay, Oxford, Clarendon Press, 1936.

6 Locke, "Carta al Dr. Thomas Molyneux", 1-XI-1692: Correspondence, vol. 4, 1979, p. 563.

7 Locke, "Carta a Nicolas Toinard" 16-VII-1678: Correspondence, vol. 1, 1976, pp. 594-5. algunos cuerpos enfermos como los conejos y los pollos bien aderezados para alimentar a otros que están sanos, aunque quizás pueden encontrarse algunas constituciones con las cuales incluso esa clase de dieta saludable no se acomodará del todo. Pero aun así, a cualquiera que haya traído la cura de alguna enfermedad a un grado de seguridad cercano al que tiene la alimentación de un hombre sano con cualquier tipo de carne saludable, puede permitírsele ser confiado en su empeño de haber perfeccionado el arte de la medicina en esa parte y para esas especies de trastorno, aunque quizás en algunos casos pertinaces e irregulares su método bien constituido pueda fallarle, y la enfermedad pueda frustrar el habitual éxito de sus empresas. Y a un grado tal de perfección como ése yo creo que puedo afirmar confiadamente que el arte de la medicina ha llegado en muchas enfermedades que raramente se resisten a los hábiles intentos de los buenos prácticos. Y no permitamos que la malicia de las personas prejuiciadas sugiera aquí que en estas promesas seguras de salud no puede confiarse más que en enfermedades tales que por sí mismas nos dejan, donde la naturaleza trabaja comúnmente por la curación sin la asistencia del arte y puede sospecharse con razón que el paciente debió su recuperación más al vigor de su propia constitución que a las drogas del farmacéutico, algunas enfermedades como algunas semillas.... (FIN DEL MANUSCRITO)

8 Locke, "Of human understanding: The Epistle to the Reader": The Works, vol.1, p. L

9 Locke, Essay on human understanding, vol. 3, B.4., cap. 12, S. 10, p. 86.

10 Bacon, Francis (1985), La Gran Restauración. «Novum Organum", Madrid, Alianza, p.159.

11 Boyle, Robert (1680). The Sceptical chymist (2a ed.) "Experiments and notes", The Authors Preface. Oxford. (Traducción castellana: El químico escéptico, Barcelona, Crítica, 2012).

12 Locke, Essay on Human Understanding, vol. 3, B.4., cap. 12, S.5, p. 82.

13 Hipócrates, "De l'ancienne médecine”. Opera Omnia (Littré), Amsterdam. Adolf M. Hakkert, 1962, vol.1, p. 605

14 Sydenham, Thomas (1848), The Works of Thomas Sydenham, R.G. Latham (tr.), Londres, The Sydenham Society, vol.1, "The Preface", p. IX.

15 Locke, Essay on Human Understanding”, vol.1, B.1, cap. 1, S. 2, p. 2. 
16 Bacon, La Gran Restauración, pp. 368-9.

17 Sydenham, "Smallpox, 1669", publicado por Kenneth Dewhurst en: Dr. Thomas Sydenham, p. 111-2.

18 Locke, "Carta al Dr. Thomas Molyneux", 20-I-1693: Correspondence, vol. IV, p. 629.

19 Locke, "Carta a Edward Clarke" 21-XII-1686: Correspondence, vol. III, 1978, p. 89.

20 Locke, "Carta a John Wynne" 8-II-1695: Correspondence, vol. V, 1979, p. 266.

21 Locke, Essay on Human Understanding, "The Epistle to the Reader" vol. 1, p. L.

22 Guenellon, Dr. P., "Carta a Locke" 9-VII-1700: Correspondence, vol. VII, 1982, p. 105.

23 Locke, Essay, col. 3, B.4, cap. 21, S. 4, p. 175.
24 Locke, Essay, vol. 3, B.4, cap. 21, S. 4, p. 176.

25 Sydenham (1848), The Works of TS, vol. 1, p. 102.

26 Locke, Essay, vol. 2, B.4, cap. 3, S. 22, p. 373.

27 Locke, Essay, vol. 3, B.4, cap.16, S. 3, p. 102.

28 Sydenham, The Works of TS, p. 40.

29 Locke, Essay, vol. 2, B.3, cap. 6, S. 9, pp. 213-4

30 Locke, Essay, vol. 3, B.4, cap. 17, S. 12, pp. 110 y 112

31 La primera edición, modernizada, de este manuscrito fue realizada por Fox Burne en 1876 en su: The Life of John Locke, pp. 222-227. Posteriormente hubo otras ediciones, como la de Alexander Gibson de 1933: The physician's art, Oxford, Clarendon Press, pp. 13-26. Y la de Kenneth Dewhurst en: Dr. Thomas Sydenham (1624-1689) His Life and Original Writings, Berkeley, University of California Press, 1966, pp. 7984. Se ofrece a continuación su primera traducción al castellano.

Harrison, J. \& Laslett, P. (1965), The Library of John Locke, Oxford, The Oxford University Press.

Hipócrates, (1962) "De l'ancienne médecine". Opera Omnia (Littré), Amsterdam. Adolf M. Hakkert.

Kretzmann, N. (1981), "La tesis principal de la teoría semántica de Locke". En: Locke y el entendimiento humano, I.C. Tipton (ed), México, Fondo de Cultura Económica, pp. 225-256.

Laín Entralgo, Pedro (1961), La historia clínica, Barcelona, Salvat.

Locke, John (1823), The works of John Locke, in ten volumes. London, printed By Thomas Tegg. Reprinted in 1963, in Aalen, by Scientia Verlag.

Meynell, Guy (1994), Locke as author of Anatomia and De arte medica, York, University of York.

Meynell, Guy (2006), "John Locke and the Preface to Thomas Sydenham's Observationes Medicae", Medical History, 50. pp. 93-110.

Romanell, Patrick (1958), "Locke and Sydenham: A Fragment on Smallpox (1670)" Bulletin of the History of Medicine, 32, pp. 293-321.

Romanell, Patrick (1984), John Locke and Medicine. A new Key to Locke, Prometheus Books, Nueva York.

Sánchez González, Miguel Ángel (1986), “Especie morbosa y filosofía empirista en el siglo XVII”, Asclepio, 38, pp.159-176.

Fox Burne, Henry Richard (1969), The Life of John Locke, Aalen, Scientia Verlag, (Reimpr. de la edición de Londres, (1876). 
Sánchez González, Miguel Ángel (1987), La presencia de la Medicina en la obra de John Locke, Madrid, Editorial de la Universidad Complutense.

Sánchez González, Miguel Ángel (1988) "Las ideas antianatómicas y antimicroscópicas de Thomas Sydenham", Asclepio 4 40, pp.223-63.

Sánchez González, Miguel Ángel (1990), “Medicine in John Locke’s Philosophy", The Journal of Medicine and Philosophy, 15, pp. 675-695.
Sánchez González, Miguel Ángel (2009), Thomas Sydenham y John Locke: Ensayo sobre la anatomía, Oviedo, KRK.

Sydenham, Thomas (1848), The Works of Thomas Sydenham, Latham, R.G. (tr.), London, The Sydenham Society.

Walmsley, Jonathan Craig, (1998), John Locke's Natural Philosophy, Thesis for the degree of Doctor of Philosophy, London, King`s College. 